Cahiers $d u$ MONDE RUSSE

\section{Cahiers du monde russe}

Russie - Empire russe - Union soviétique et États indépendants

$46 / 4 \mid 2005$

L'invention d'une politique humanitaire

\title{
Yaacov Ro'i, ed., Democracy and Pluralism in Muslim Eurasia
}

\section{Sébastien Peyrouse}

\section{(2) OpenEdition \\ Journals}

Édition électronique

URL : https://journals.openedition.org/monderusse/6659

DOI : 10.4000/monderusse.6659

ISSN : $1777-5388$

Éditeur

Éditions de l'EHESS

Édition imprimée

Date de publication : 1 décembre 2005

Pagination : 966-968

ISBN : 2-7132-2057-2

ISSN : $1252-6576$

\section{Référence électronique}

Sébastien Peyrouse, "Yaacov Ro'i, ed., Democracy and Pluralism in Muslim Eurasia », Cahiers du monde russe [En ligne], 46/4 | 2005, mis en ligne le 12 septembre 2013, consulté le 03 septembre 2022. URL : http://journals.openedition.org/monderusse/6659 ; DOl : https://doi.org/10.4000/ monderusse. 6659

Ce document a été généré automatiquement le 3 septembre 2022.

Tous droits réservés 


\title{
Yaacov Ro'i, ed., Democracy and Pluralism in Muslim Eurasia
}

\author{
Sébastien Peyrouse
}

\section{RÉFÉRENCE}

Yaacov Ro'I, ed., Democracy and Pluralism in Muslim Eurasia. Londres, New York :

Frank Cass, 2004, 403 p. (Cummings Center Series, 19)

1 L'objectif de cet ouvrage collectif dirigé par Yaacov Ro'i, spécialiste de l'islam soviétique et auteur de plusieurs autres livres, est de proposer une analyse détaillée de la mise en place d'institutions démocratiques dans les zones de tradition musulmane de l'espace postsoviétique. Une des interrogations communes à l'ensemble des dix-neuf contributions, d'auteurs occidentaux ou postsoviétiques, est de savoir si le substrat musulman peut être retenu comme l'un des facteurs d'analyse pertinents pour comprendre les évolutions politiques de ces nouveaux États. Le livre s'appuie en effet sur le postulat selon lequel les sociétés ne peuvent évoluer toutes vers la démocratie selon des modalités uniformes. L'une des autres composantes à prendre en compte est le poids du legs soviétique, qui pourrait, dans un contexte politique et économique complexe, jouer en défaveur du principe démocratique.

2 La première partie fait le point sur "l'arrière-fond historique» de la question en consacrant quatre articles à la période précoloniale et coloniale. Les deux premiers (de Zhanylzhan Dzhunusova sur le Kazakhstan et de Moshe Gammer sur le Daghestan) s'intéressent aux structures politiques des sociétés précoloniales. Comme le montrent les auteurs, une meilleure connaissance des formes de démocratie qui ont pu exister dans ces proto-États permet d'éclairer les symboliques qui, remises au goût du jour, sont aujourd'hui présentées comme les précédents de la démocratie nationale. Les deux articles suivants (de Azade-Ayse Rorlich sur la question de l'appartenance identitaire des musulmans de Russie et de Dov Yaroshevski sur le khanat de Khiva) s'interrogent sur l'impact de la présence russe et les transformations politiques subies par ces sociétés 
dans les dernières décennies $\mathrm{du} \mathrm{XIX}^{\mathrm{e}}$ et les toutes premières $\mathrm{du} \mathrm{Xx}^{\mathrm{e}}$ siècle : les expériences démocratiques qui ont suivi la révolution de 1905 ont en effet rendu possible une première organisation politique des musulmans de l'empire et y ont suscité une réflexion interne sur le constitutionnalisme.

La seconde partie aborde plus précisément les " points de tension potentiels » avec deux contributions, l'une de Richard Pomfret sur l'impact de l'aide économique occidentale, et l'autre de Yaacov Ro'i sur la place de l'islam dans l'établissement de logiques démocratiques. Considéré par les gouvernements en place comme la principale menace pour leur pouvoir, l'islam se voit accusé de remettre en cause les acquis de la sécularisation soviétique comme ceux de la nouvelle démocratie, tout en subissant, de la part des autorités, de violentes atteintes au principe démocratique. Les deux dernières parties, les plus longues, sont consacrées à la situation contemporaine, l'une dans les États indépendants d'Asie centrale et du Caucase, l'autre dans les régions musulmanes de la Fédération de Russie. La première consacre un article à chaque État d'Asie centrale (deux au Kirghizstan) à l'exception du Turkménistan, et un à l'Azerbaïdjan. Les auteurs s'y interrogent sur les espoirs de démocratie suscités par l'accession à l'indépendance et sur la manière dont chaque État a tenté de gérer, sur un mode à chaque fois spécifique, les mêmes défis politiques, économiques et sociaux. Tous constatent aujourd'hui combien la démocratie reste encore de façade, les institutions démocratiques ne jouant pas réellement leur rôle, en raison notamment de la concentration des pouvoirs aux mains du clan présidentiel et de la mise en œuvre de politiques radicales au nom du maintien de la stabilité.

L'article de Neil Melvin examine par exemple les divers rythmes de passage à l'autoritarisme qu'ont connus trois États d'Asie centrale : l'Ouzbékistan, le Kazakhstan et le Kirghizstan, et tire un bilan à court terme plutôt négatif sur leurs possibles évolutions. Vladimir Babak s'attache plus spécifiquement aux partis politiques et démontre combien un pluralisme de façade, loin de témoigner d'une quelconque pratique démocratique, révèle plutôt l'inefficacité de telles structures face à la concentration extrême des pouvoirs. Pal Kostoe se focalise, quant à lui, sur un sujet important et trop peu souvent pris en compte : l'instrumentalisation de la question ethnique à des fins de durcissement politique. Il analyse à ce titre comment les stratégies mises en œuvre par le président kazakh Nursulan Nazarbaev à l'encontre de la minorité russe lui ont assuré une plus grande assise sociale. Vladimir Mesamed part du même postulat en démontrant que le président ouzbek Islam Karimov a tenté de compenser son autoritarisme en garantissant à la population ouzbek une politique résolument ethnocratique et linguistique en sa faveur. Deux articles étudient ensuite la situation, souvent présentée comme spécifique, du Kirghizstan, et son image, dans les années 1990, d'îlot démocratique en Asie centrale. Le premier, d'un ancien conseiller du président Askar Akaev, démis en mars 2005, use de la langue de bois pour décrire la situation du pays, tout en reconnaissant en partie les égarements politiques des dernières années de la présidence d'Akaev. Le second, de Vladimir Khanin, dresse un bilan contrasté des réalités kirghiz et met en relief le rôle fondamental du système de clans en tant qu'institution de régulation sociale. Il en va de même des articles de Saodat Alimova sur le Tadjikistan et de Farda Asadov sur l'Azerbaïdjan, qui présentent une analyse fine des situations respectives de ces pays, le premier n'ayant pas réussi à poursuivre le processus démocratique qui avait émergé des accords de paix de 1997, tandis que le second, malgré 
l'existence d'une opposition constituée, reste aux mains de la famille présidentielle Aliev.

5 La quatrième et dernière partie est consacrée à diverses entités de la Fédération de Russie : le Tatarstan (Rafik Muhametshin), le Bachkortostan (Ildus Ilishev), le Daghestan (Enver Kisriev), le Nord-Caucase dans son ensemble (Svante Cornell) et le cas à part de la Tchétchénie (Alexei Kudriatsev). Si les enjeux y sont diversifiés - certaines républiques ayant su trouver un modus vivendi avec le centre moscovite, d'autres étant en conflit avec lui -, les défis à relever restent semblables : arriver à concilier sentiment national et appartenance à l'entité fédérale russe, accorder à l'islam une certaine place dans la vie publique sans remettre en cause la laïcité officielle du pays et la supériorité numérique des orthodoxes, créer des structures démocratiques représentatives de la collectivité au niveau républicain, tout en participant à la vie politique de la Fédération dans son ensemble.

6 On ne peut donc que saluer la publication de ce livre et souligner son importance dans le domaine de la recherche sur l'Asie centrale et plus généralement le monde musulman postsoviétique. L'attention portée non seulement à l'héritage soviétique mais également au passé tsariste de la région évite par exemple nombre d'erreurs et de clichés véhiculés dans certains ouvrages qui n'ont pas le recul historique nécessaire. Si le bilan des années d'indépendance présenté dans le recueil reste critique en ce qui concerne le processus démocratique, il n'en demeure pas moins objectif et nuancé. Les auteurs ont en effet su mettre en avant les difficultés rencontrées par ces jeunes États sans occulter le rôle des élites politiques en place dans les échecs et les durcissements autoritaires en cours. Cette approche approfondie de la zone devrait permettre au livre de devenir un classique en la matière et débouchera sans doute sur de nouvelles recherches. 